\title{
Relatos disidentes sobre experiencias de maternidad. Una lectura desde el punto de vista feminista de las escrituras en primera persona que circulan en plataformas digitales. Los casos de: "Mamá Mala" y "Las dos mamis, activismo virtual"
}

\author{
Eva Rodriguez Aguero \& Magalí Cingolani \\ Universidad Nacional de Cuyo, Argentina \\ E-mail: evarodriguezaguero@gmail.com/magali.cingolani@gmail.com
}

\begin{abstract}
Resumen
Este trabajo se ocupa de analizar la presencia en plataformas digitales de relatos en primera persona que abordan experiencias vinculadas a la maternidad desde un punto de vista crítico en relación a los mandatos y expectativas que plantean los imaginarios sociosexuales dominantes. Para las mujeres, lesbianas, travestis y trans, Internet ha significado una potente herramienta para pensar y narrar el mundo desde una mirada propia. Algunas autoras que se inscriben en el ciberfeminismo (Velásquez Herrera, 2015; Zafra, 2008; Haraway,1985) consideran que la posibilidad de socializar esas formas otras de narrar, de un modo des-centrado en relación a la visión

masculina, heterosexual, blanca y occidental -y sin mediación del saber profesional, de la academia o la industria editorial- no sólo ha tenido consecuencias positivas para las mujeres y las identidades disidentes, sino que ha transformado las dinámicas de circulación y acceso a saberes y posicionamientos no hegemónicos. Partiendo del punto de vista de la epistemología feminista, que concibe a todo conocimiento como situado (Haraway, 1985) se recurre a una metodología basada en el análisis semántico de contenidos para abordar un corpus compuesto por: el perfil de Facebook "Mamá Mala" (Argentina) y el blog "Las dos mamis, activismo virtual" (Méjico).

Palabras clave: feminismos; escrituras; maternidad; plataformas digitales.
\end{abstract}

\section{Dissenting narrations about motherhood experiences. A reading from the feminist point of view of personals writings that circulate in digital platforms. The cases of: "Mama Mala"and "Las dos mamis, Activismo Virtual".}

\begin{abstract}
This document analyze the presence in digital platforms of first-person narrations that approach experiences related to motherhood from a critical point of view, in relation to the mandates and expectations of the dominant socio-sexual imaginaries. For wo-

men, lesbians, transvestites and trans, Internet has meant a powerful tool to think and narrate the world from a glance of their own. Some authors who enroll in cyberfeminism (Velásquez Herrera, 2015, Zafra, 2008, Haraway, 1985) consider that the possibi-
\end{abstract}

Data de submissão: 2018-03-31. Data de aprovação: 2018-04-26.

A Revista Estudos em Comunicação é financiada por Fundos FEDER através do Programa Operacional Factores de Competitividade - COMPETE e por Fundos Nacionais através da FCT - Fundação para a Ciência e a Tecnologia no âmbito do projeto Comunicação, Filosofia e Humanidades (LabCom.IFP) UID/CCI/00661/2013.
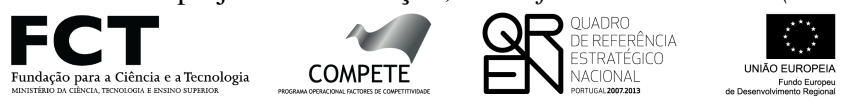

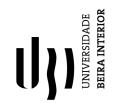

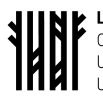

Estudos em Comunicação no 27, vol. 1, 127-141

Dezembro de 2018 
lity of socializing these other forms of narration, in a de-centered way in relation to the masculine, heterosexual, white and occidental vision - and without mediation of professional knowledge, academia or the publishing industry - has not only had positive consequences for women and dissident identities, it has also transformed the dynamics of circulation and access to non-hegemonic knowledges and positio- nings. Starting from the point of view of the feminist epistemology, which conceives all knowledge as situated (Haraway, 1985), we resort to a methodology based on semantic content analysis to approach a corpus composed of: the Facebook profile "Mama Mala"( Argentina) and the blog "Las dos mamis, virtual activism"(Mejico).

Keywords: feminisms; writings; motherhood; digital platforms.

$\mathrm{E}^{\mathrm{L}}$

L presente trabajo se propone reflexionar sobre la politización de las experiencias de maternidad, por parte de algunas mujeres y disidentes sexuales a partir de la puesta en circulación de narrativas en primera persona, a través de posteos en redes sociales y blogs.

Se parte de la idea que las redes sociales se han constituido en los últimos años en una especie de tribuna, a partir de la cual las mujeres -y otros grupos subalternos- han tomado la palabra, en pos de difundir ideas y prácticas contra hegemónicas que no hallaban lugar de enunciación en otros espacios (Velázquez, 2015; Manzanares, 2015; Reguillo, 2017). De un tiempo a esta parte, estas redes han comenzado a operar como una eficaz herramienta para la difusión de aquellos posicionamientos políticos que luchan contra el racismo, la opresión de clase, el extractivismo o la homolesbotransfobia.

Las activistas ciberfeministas detectaron tempranamente esta potencialidad de las redes sociales y evolucionaron, desde los primeros ciberfeminismos artísticos al ciberfeminismo social que, según Boix (2007), tiene como objetivo difundir información, generar redes de activismo y solidaridad, coordinar iniciativas para mejorar de manera concreta la vida de las mujeres, además de servir de freno a las acciones de abuso de un patriarcado también globalizado.

Así, las redes sociales y su uso extendido abrieron un mundo de posibilidades diferentes dentro de la web, convirtiéndose en la principal herramienta del activismo en redes.

Actualmente, fuera de la égida de influencia de las modas editoriales, de la homogeneización que proponen los medios hegemónicos o el circuito de revistas especializadas, es decir, fuera de toda institucionalidad normalizadora, algunas mujeres que transitan experiencias de maternidad utilizan las plataformas y redes sociales como vehículo para compartir relatos en primera persona sobre las mismas. Así, desde una mirada que busca descolonizar y despatriarcalizar estas vivencias, dichos relatos proponen comenzar a vivirlas, pensarlas y narrarlas fuera de lo que la psicoanalista Ana María Fernández (1994) llama los "mitos sociales de la maternidad". Mitos bajo los cuales, en Occidente, se ha estructurado el deber ser en relación a esta poderosa experiencia.

Estos relatos -habida cuenta del valor político que adquieren las experiencias personales con su puesta en circulación en el espacio público- van a contrapelo de los conceptos normativos en torno a los roles de género, presentes en los discursos médico, legal, escolar, religioso y mediático (Scott, 1993).

Un rastrillaje a contrapelo de la Red nos arroja interesantes hallazgos, pues se percibe la emergencia de no pocos sitios en los que se aborda el tema de la maternidad, desde una mirada crítica en relación con los modelos dominantes. Es cierto que los relatos dominantes (tanto en publicaciones 
destinadas a las mujeres-madres, como en las interacciones personales en redes sociales) siguen siendo aquellos que se ajustan a los mandatos tradicionales pero, en paralelo, es claramente perceptible la presencia de este otro tipo de discursos que podríamos llamar emergentes ${ }^{1}$ (Williams, 1980). Algunos ejemplos son los blogs:

Mujer, madre y argentina (http://mujermadreargentina.com.ar/blog/); Libertad Condicional (https://libertadcondicionalblog.wordpress.com/); Soy la Zoila (www.soylazoila.com) y Club de Malas Madres (https://clubdemalasmadres.com). Por su parte, sobre lesbomaternidades se cuentan: Ella, los trillizos y yo (http://ellalostrillizosyyo.blogspot.com.ar/); La suma de Nosotras (http://lasumadenosotras-aquaylotus.blogspot.com.ar/); Mamás Lesbianas y Bebé (www.mamasle sbianasybebe.com/); Mamis por dos. Madres Lesbianas (http://mamispordos.blogspot.com.ar/); Dos lesbianas, 9 meses y una nueva Vida (http://mamaymama.blogspot.com.ar/); Dos mujeres, un niño, y lo que venga (http://arrietayfamara.blogspot.com.ar/) y Una familia diferente, la mía; (http://unafamiliadiferentelamia.blogspot.com.ar/).

También los perfiles de Facebook: Mamá sobre Ruedas y Mamá Mala.

En este trabajo se ha optado por analizar dos de los anteriormente mencionados: el perfil de Facebook de Carolina Justo Von Lurzer y su personaje Mamá Mala y el blog Las dos mamis, activismo virtual (http://dosmamis.blogspot.com.ar/).

Vigilantes ante los riegos políticos que implican los discursos celebratorios o acríticos sobre las Tecnologías de la Información y la Comunicación (TICs) se parte del supuesto de que, en las redes, y en medio del tupido bosque que constituyen prácticas como el ciberacoso, la difusión de imágenes estereotipantes o los discursos antifeministas, en la redes las mujeres están haciendo oír sus voces rebeldes.

Por otra parte, resulta relevante destacar que no todas las mujeres se hallan en igualdad de condiciones para producir este tipo de narrativas. Es cierto que el acceso a la escritura y a Internet sigue siendo un privilegio en nuestros países del Sur. Es cierto que la brecha de género y de clase atraviesa también la estructura y el funcionamiento de los intercambios en las redes.

Aun a sabiendas de estos aspectos, consideramos que no es menos cierto que, de un tiempo a esta parte, Internet se ha transformado, para las mujeres de sectores medios que se comunican por redes sociales, en una potente punta de transgresión, capaz de resquebrajar las jerarquías patriarcales que oscurecen las posibilidades de inscribir las historias y experiencias de las mujeres y el colectivo LGTTTBIQ ${ }^{2}$, en la arena de los discursos públicos.

Por otro lado, es importante destacar que esa verdadera "cultura feminista" que tiene lugar en las redes, no ha implicado una omisión o una disolución de los cuerpos, de las cuerpas, rebeldes. Pues desde muchas de estas plataformas se agita un activismo que no se desvanece en el ciberespa-

1. Junto con Williams (1980) entendemos por emergente a aquellas nuevas prácticas culturales, nuevos significados y valores, nuevas relaciones y tipos de relaciones que se crean continuamente. Se trata de elementos que, eventualmente, pueden ser incorporados o destruidos por la cultura dominante, teniendo en cuenta que ésta deja intersticios para que surjan elementos residuales o emergentes. En esta dirección, toda práctica cultural puede entenderse en relación con lo dominante.

2. Son las siglas que identifican a las palabras lesbiana, gay, bisexual, transgénero, travesti, transexual, intersex, queer y además es un movimiento que se conformó por la lucha de los derechos de igualdad para estas comunidades sexuales disidentes. 
cio, sino que por el contrario, encuentra su correlato en las manifestaciones callejeras. De hecho, muchas de las narraciones en primera persona que por allí circulan, giran en torno a experiencias ancladas al cuerpo. Tal es el caso de los relatos en clave anti heteropatriarcal, de las experiencias de maternidad, "la reina de las experiencias", al decir de Adrienne Rich (1986).

Cabe señalar que si bien este trabajo claramente no es un estudio sobre la maternidad, al ser ésta el objeto de las narraciones sobre las que se hace foco, es importante señalar que entendemos a la misma a partir de una mirada crítica que recurre a las teorizaciones realizadas desde los estudios de género y la teoría feminista. Teniendo en cuenta que, si en nuestra cultura, las concepciones, ideas y prácticas ligadas a la maternidad son comprendidas a partir de la construcción de un cierto sentido común que la entiende como un hecho estrictamente ligado a la naturaleza, que nada tiene de cultural y que por lo tanto es fijo e inmutable a las transformaciones sociales, gracias a los aportes de la teoría feminista sabemos que los sentidos que se le asignan socialmente, forman parte de una serie de complejas construcciones simbólicas que se fueron forjando a lo largo de la historia, asociando a las mujeres con determinadas funciones y valoraciones, naturalizadas a partir de argumentos biologicistas.

La cultura occidental moderna ha planteado un modelo hegemónico de madre. Éste está construido sobre un imaginario que entiende a la maternidad como una actividad de dedicación exclusiva y de realización personal para las mujeres, dejando de lado las particularidades y contextos específicos en que éstas se insertan, así como su carácter de construcción social. Partiendo de esta idea, Ana María Fernández (1994) sostiene que la maternidad es una función social que ha sufrido transformaciones a lo largo de la historia, y cuya concepción se relaciona íntimamente con las condiciones materiales, y el modelo de desarrollo (político y económico) presente en cada contexto. De hecho, desde nuestra perspectiva, la emergencia de nuevos relatos, acerca de la misma, no pude explicarse como una transformación que se da en el campo de lo meramente discursivo. Sino, que más bien, la emergencia discursiva de formas "otras" de experimentar y narrar la maternidad, son el correlato de transformaciones profundas que atraviesan a nuestra sociedad, y que encuentran su base material en la creciente visibilización de sexualidades disidentes, el surgimiento de nuevos imaginarios sociales en torno a éstas y la conquista - gracias a la lucha de los/las/les activistasde marcos legales mas inclusivos y menos hetenormativos en relación a los roles de género y las familias.

\section{LA MATERNIDAD COMO EXPERIENCIA}

Adrienne Rich (1986) distingue entre ésta como institución y como experiencia. La maternidad como experiencia hace referencia a la relación potencial de cualquier mujer con los poderes de la reproducción y con los hijxs. Mientras que la maternidad como institución implica que ese potencial y sus protagonistas, las mujeres, permanezcan bajo el control patriarcal. Al hablar de la maternidad como institución, se hace referencia a los medios con los que el poder se mantiene y transfiere, y a los entramados invisibles que garantizan que ese poder quede en determinadas manos (Rich, 1986).

Puesto que el cuerpo de las mujeres y sus capacidades reproductivas representan un gran valor para toda sociedad que pretenda perpetuarse y que sólo las mujeres poseemos un cuerpo que pro- 
duce otro cuerpo, en pos de asegurar un control efectivo de la reproducción, los saberes científicos han buscado apropiarse de las capacidades sexuales y reproductivas de las mujeres, naturalizándolas como una obligación o condición biológica y trastocando el poder en subordinación (De Barbieri, 1993).

La patologización de la diferencia, la medicalización del embarazo y el parto, la violencia obstétrica y la asociación inmediata de las figuras de mujer y madre son algunos de los factores que permiten que esta apropiación se lleve a cabo. Cabe señalar que esta apropiación implica también la expropiación de los relatos sobre estas experiencias a las mujeres, y su colonización por parte los saberes masculinos.

La maternidad como institución cuenta con una serie de medidas que permiten que se perpetúe, tales como las leyes que regulan los nacimientos y el aborto, la negativa a considerar las tareas domésticas como parte de la producción, la ausencia de atenciones sociales a las madres, el confinamiento solitario de la maternidad y la naturaleza simbólica de la paternidad, que da a los varones derechos y privilegios sobre los, las hijos/as, frente a los cuales asumen -en muchos casos- responsabilidades mínimas.

Por el contrario, la maternidad como experiencia, refiere a la serie de prácticas y vivencias diversas en torno al nacimiento, crianza y vínculo de las mujeres con sus hijxs, y que por lo tanto implican diferentes caminos en la construcción de las identidades de esas sujetos madres. Concebida así, se habilitan múltiples modos de ser madre: soltera, lesbiana, indígena o incluso asumir dicha función de manera colectiva. Es decir, distintas formas de adaptarse a situaciones sociales y económicas, de relacionarse con el propio cuerpo y por, lo tanto, de ser madre.

En los siguientes dos apartados nos abocaremos al análisis de casos, a partir de los cuales dos mujeres madres narran en la web sus experiencias en primer persona. Del universo de blogs y sitios localizados -y dada la necesidad de ajustarse a determinada extensión- seleccionamos como corpus a trabar en este artículo un perfil de Facebook y un blog. Ambos pertenecen a mujeres de sectores medios, con acceso a la instrucción, y que -desde su particular visión- construyen narrativas sobre sus propias experiencias de maternidad, inscribiéndolas en el espacio público, vía la utilización de redes sociales.

En cuanto a la metodología utilizada se tomaron los aportes metodológicos de la etnografía virtual, definida por Hine (2004) como una herramienta que problematiza el uso de Internet y adapta las estrategias de la etnografía clásica al estudio de las interacciones en la red (etnografía online), tomando en cuenta además, las realidades concretas y los contextos desde los cuales los/as usuarios/as interactúan (etnografía offline).

Además, se recurre al análisis semántico de contenido. A partir de éste, se define cierta estructura significativa de relación, y se consideran todas las ocurrencias que concuerden con dicha estructura (Andreu Abela, 2011). El análisis por categorías consiste en distinguir, separar y priorizar elementos en los discursos a estudiar para reconocer los tópicos o lugares comunes presentes en los mismos, definiendo los patrones de relación a tener en cuenta a partir de las teorizaciones sobre el tema (Echeverría, 2005). En este caso, son de especial relevancia las nociones planteadas desde la teoría por Rich (1986) acerca de la recuperación de la maternidad como experiencia, en tanto que re apropiación de las vivencias personales, por fuera de los saberes "oficiales". Por 
otra parte, la idea de que existen mitos sociales dominantes acerca de la maternidad, trabajada por Fernández (1994) es el núcleo en torno al cual ambos relatos giran en pos de decosntruirlos.

\section{Cómo SER Una Mamá mala}

La posibilidad de ser una madre que transgrede los mandatos heteropatriarcales que colocan al amor materno -basado en la existencia de un supuesto instinto maternal- en la cúspide de las virtudes morales que un ser humano puede encarnar, es el desafío que toma Mamá Mala. El personaje creado por Carolina Justo Von Lurzer $^{3}$ (JVL) narra, a través de posteos en la red social Facebook, las experiencias del puerperio que ésta atraviesa, luego de la llegada de su segundo hijo, en 2014, a partir de lo cual, según ella misma describe, experimentó sensaciones que no había vivido con el nacimiento del primero. A partir de esto creó Mamá mala, un personaje de ficción que le permitía decir aquellas cosas que a una buena madre generalmente le están vedadas desde el punto de vista moral.

Si bien JVL, es universitaria y especialista en estudios de género, sus relatos no buscan ser reflexiones teóricas sobre la maternidad, se trata más bien de intervenciones en el registro del género "diario íntimo", registro habitualmente usado entre quienes utilizan esta red social, aun a sabiendas de que dichos escritos -vía su publicación- serán de acceso público en la web. Y no sólo eso, sino que -dada la particularidad de la plataforma- eventualmente serán objeto de opiniones y respuestas por parte de los/as usuarios/as con los/as que interactúa en la red social.

Sus estados ${ }^{4}$ en Facebook se caracterizaron por estar escritos en clave de humor y cuestionar los mandatos sociales vinculados a la maternidad desde el relato de situaciones y vivencias personales.

Si la buena madre es la encarnación del deber ser; un deber ser que sintetiza el sumun de la entrega personal y el vivir para otros y otras (entiéndase, familia, hijos e hijas), por el contrario, nuestra cultura reserva para la mala madre la oscura contracara de la primera.

Por otra parte, los posteos de JVL se insertan en el contexto vital del puerperio. El mismo -en líneas generales- es un tema cercado por silencios de diversa índole. Principalmente, silencios en cuanto a las experiencias particulares de cada mujer-madre, pues la escasa información que suele circular al respecto es producto de las elaboraciones realizadas por parte de los saberes legitimados: medicina, psicología, derecho.

En nuestra cultura, los avatares de este singular período tampoco suelen ser demasiado abordados entre pares. La idealización de la maternidad como terreno de dicha y gozo en el cual las mujeres "naturalmente" se desenvuelven con soltura y sin contradicciones, dificulta la existencia

3. Carolina Justo V.L. es argentina, Doctora en Ciencias Sociales y Magíster en Comunicación y Cultura, es también Docente en el Profesorado en Ciencias de la Comunicación de la Facultad de Ciencias Sociales y en el Seminario de Cultura Popular y Cultura masiva de la Carrera de Comunicación de la UBA. Es investigadora asistente en el CONICET, y sus investigaciones se desarrollan en torno a los imaginarios sociosexuales y de género en y a partir de los medios masivos de comunicación.

4. Un "Estado" de Facebook es una publicación o comentario que aparece en la propia biografía, visible para los usuarios "amigos" del perfil o para todos, según las condiciones de privacidad que se establezcan. Cada estado da cuenta del día y la hora en el que fue escrito (Luna, 2013). 
de un espacio en el cual haya lugar para las dudas, el temor o la ambivalencia de sentimientos en cuanto al hijo/hija y en cuanto a la experiencia vital que se atraviesa.

JVL hace de este tema una rica cantera, a partir de la cual extrae experiencias a menudo concebidas como relativas al ámbito privado. A partir de su puesta en palabras, se permite liberarlas del encierro forzoso entre las cuatro paredes de la habitación, a la que han sido históricamente confinadas, para hacerlas jugar en la arena de los discursos públicos.

$\mathrm{Si}$ en las sociedades occidentales este período funciona como un paréntesis en la vida productiva de las mujeres de los sectores medios, vía licencias laborales y suspensión de muchas de las rutinas cotidianas; en el caso de JVL, la red social Facebook se convierte en la vía de escape a partir del cual transcender ese "retiro", muchas veces no elegido, y poder dar cuenta públicamente de los avatares de la vida diaria durante los meses posteriores al parto.

El malestar ante la situación de aislamiento y frente al hecho de no encajar en el molde que prevén los mandatos sociales (en tanto se supone que una puérpera sólo puede tener sentimientos de amor, ternura y compresión hacia su hijo/a) es expuesto aquí en términos políticos. De hecho, desde esta plataforma comparte sentimientos que por momentos develan, incluso, la no empatía para con su hijo, algo impensado en las narrativas hegemónicas sobre la maternidad.

Otro de los lugares comunes de la maternidad contra el cual JVL arremete, es la operación a partir de la cual se ubica a la madre en segundo plano, dejando al niño o la niña y sus cuidados como centro exclusivo de atención durante ese período.

JVL produce en su primer comentario ${ }^{5}$ una transgresión en relación a esto. El relato autocentrado la posiciona en un primer plano, por delante del niño, una constante que veremos repetirse a lo largo de las subsiguientes publicaciones. De ahí en más, hablará en primera persona acerca de sus sentires, autocosntituyéndose como protagonista de esa experiencia.

Así, a partir de la escritura de esas vivencias, "Mamá Mala" se lanza a cruzar el océano del puerperio.

Dice en su muro:

Ayer me decidí a combatir la depresión posparto. Me calcé al muchachito, hicimos trámites para él y para el mayor, me senté en La Cigüeña a comer nuestro primer super de pollo juntos y observé con satisfacción que la soledad del puerperio se puede atravesar en esa distante compañía de los desconocidos de bar. Hoy, abro los mails y me pregunto qué me hará mejor en un día de lluvia, si 12 sesiones de ultracavitación o 12 piezas de Sushi. Mientras tanto, Felipe me habla en gorgoritos... menos sola que ayer.

A partir de la lógica que impone esta red, la escritura de posteos genera la interacción con otros/as sujetos. Así, las seguidoras -en su mayoría mujeres- dan "me gusta", colocan emojis o "comentan" el posteo. En ese intercambio virtual (que le otorga a esos escritos una particularidad especial, que rompe el aislamiento) surge otro de los temas a partir del cual JVL se posiciona desde un discurso disruptivo, pues sostiene la no obligatoriedad o la imposibilidad de amamantar. Veamos:

5. El mismo tiene como fecha de publicación el 23/02/2014. 
Andrea Lacombe sushi sin lugar a dudas! la ultracavitación para cuando dejes de amamantar jeje 18 de febrero de 2014 a las 15:15 - Me gusta - 1

Carolina Justo amamantar, dijiste la palabra maldita. Felipe odió la teta desde el dia 1 y yo no amamanto, sólo materno, que sin amamantar es bastante menos amable 18 de febrero de 2014 a las 15:16 - Me gusta - (1) 2

Andrea Lacombe uhhh! no sabia $\because$ con más razón el sushi entonces 18 de febrero de 2014 a las 15:17 - Me gusta - 22

JVL, explicita que el hecho de no poder amamantar le genera cierta cuota de frustración. El mandato que existe en torno a la lactancia es quizás uno de los más fuertes, pues se halla socialmente construido como un elemento clave e indispensable para el desarrollo del niño/a; a la vez que alentado desde el Estado por medio de campañas públicas.

Según el modelo instituido, todas las madres pueden (y deben) dar de mamar, y -apoyándose en cierta idea de que todo lo que tiene que ver con la maternidad es del orden de lo "natural"cualquier inconveniente que puedan impedir esta función se debe a la incapacidad o a la mala voluntad de la madre, a la que se considera "desnaturalizada".

Su testimonio, bajo la forma de posteo, deja entrever cierta tensión entre la realidad de su experiencia de maternar a un recién nacido y el modelo socialmente establecido.

Se trata de un comentario que dispara sobre el núcleo duro de los mandatos maternos: maternar es igual a amamantar.

En relación a la posibilidad de romper con este binomio, ella dice: "yo sólo materno, no amamanto y maternar sin amamantar, es bastante menos amable". La búsqueda por correrse de dicha obligatoriedad pareciera generarle ambivalencia. A propósito de esto, JVL argumenta en una doble dirección: por un lado, se afirma en su imposibilidad. Pero por el otro, algunos de sus escritos expresan cierto grado de frustración por no poder hacerlo.

Se percibe también cierta tensión o puesta en cuestión en relación a los discursos "pro lactancia" de tipo progresista, los cuales (aunque desde argumentos diferentes a los de los discursos conservadores) imponen la lactancia materna como única opción para la alimentación de un recién nacido.

En otros comentarios, JVL pone en cuestión a la maternidad como fuente exclusiva de satisfacción personal para las mujeres. Veamos:

Quejarse de yyena: acabo de recibir la comunicación del inicio formal - es decir, de que al fin la guita está disponible- de un proyecto PICT que dirijo. Bajé los formularios, están llenos de palabras e indicaciones, yo estoy llena de caca y mamaderas. Voy a ir presa ${ }^{6}$.

6. Fecha de Publicación: 05/03/2014. 


\begin{tabular}{l}
\hline Me gusta $\Rightarrow$ Compartir \\
\hline 16
\end{tabular}

Ah, no, pero ahora la Facultad tiene el tupé de pedirle a mamá mala que solicite el incentivo... ¿tenés idea, Sociales, de la cantidad de incentivos que necesita mamá mala siquiera para poder incentivarse a solicitar el suyo? Por favor... ${ }^{7}$

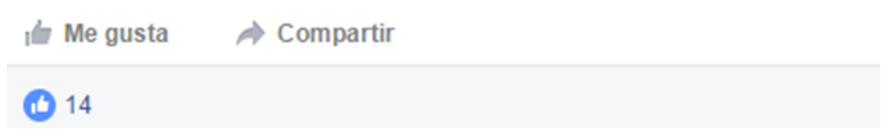

En estos estados vemos cómo la maternidad es abordada por Mamá Mala como un factor que compite -y por momentos- obstaculiza sus inquietudes intelectuales y proyectos profesionales. Si bien esta situación es vivida por muchas mujeres que depositan deseo y expectativas en su vida laboral, el tema rara vez logra permear la capa de los discursos mas instalados socialmente. Podríamos decir que esta problemática halla escasas posibilidades de decibilidad, al menos en la esfera de los discursos públicos.

Así, haciendo uso de una perspectiva crítica en torno a las relaciones de género y cuestionando lo que ella llama "desigualdades de origen", relata con humor, algunas anécdotas cotidianas en las que busca visibilizar -sin desmedro del amor que a la vez declara hacia sus hijos- que, en ocasiones, éstos representan para ella una dificultad en relación a su desarrollo profesional.

Mamá Mala, este personaje inventado, le permite a su creadora poner en palabras una serie de cosas que no entran dentro del umbral de lo que una madre pude decir en nuestra cultura. Se trata de intervenciones que siguen aquello de lo personal como político, aquello de que las experiencias personales son reflejo y síntoma de relaciones de poder que tienen lugar en el ámbito privado del hogar, pero que se inscriben y sostienen en el contexto mas amplio del entramado social.

Si bien el lugar de enunciación de la autora es crítico respecto de los mandatos sociales, también deja entrever que el hecho de intentar romper con los mismos no la desliga -de un momento a otro, como por arte de magia- de esa enorme mochila. En su caso, estas experiencias son resignificadas a partir de tomar estado público a través de la red social, dándole un sentido y una lectura política a todo aquello que es habitualmente naturalizado desde los modelos establecidos de mujer y madre.

\section{MAMÁ Y MAMÁ}

El segundo de nuestros casos de análisis es el blog Las dos mamis, activismo virtual ${ }^{8}$ y que surge en el año 2006. Sus autoras, las mejicanas Ana de Alejandro y Criseida Santos, son do-

7. Fecha de Publicación: 17/03/2014.

8. En este blog se encuentran posteos escritos por ellas, información útil para las madres lesbianas de México, y para aquellas que tengan el deseo de serlo, así como artículos periodísticos de interés. 
centes y madres lesbianas de mellizos, Decidieron involucrarse en el activismo virtual a partir del embarazo de sus hijos.

El blog es una suerte de bitácora que relata episodios íntimos sobre la forma en que las dos mujeres crían a sus hijxs.

Monique Wittig (2006) sostiene que las lesbianas no son mujeres ${ }^{9}$, sino que están más allá de las categorías (mujer y hombre), pues el sujeto designado lesbiana, no es una mujer ni económica, ni política, ni ideológicamente. Si esto es así, y si nuestra cultura busca fijar la ecuación: mujer igual a madre, una madre lesbiana, en palabras de Wittig: una no mujer, deviene en algo ciertamente disruptivo para las concepciones dominantes.

Ambas madres, a partir de narrar sus experiencias en la web, buscan expresamente visibilizar y colaborar a la legitimidad de las maternidades lesbianas, asunto que no se reduce aquí a la narración de experiencias en primera persona, sino que se extiende a un posicionamiento en pos del activismo. En 2008, en Monterrey, ambas fundan la Comunidad de Madres Lesbianas (Comales); participando activamente en la lucha por los derechos y el reconocimiento de las múltiples alternativas de conformación familiar en su país. En este sentido, el blog no debe analizarse como una excepcionalidad. Tal como señalábamos al comienzo, la web da cuenta de una notable proliferación de sitios dedicados a visibilizar las lesbo maternidades.

En Las dos mamis, los posteos abogan por una maternidad y crianza militantes. Es decir, ser una madre lesbiana es considerado por ellas como una posibilidad para poner sobre la mesa de las discusiones públicas ciertas demandas y problemáticas a menudo invisibilizadas.

Decíamos que la crianza de hijos e hijas en el marco de este tipo de familias es percibida aquí como una cuestión política o una forma de militancia, en tanto que implica, nada más y nada menos que forjar sujetxs capaces de transformar el mundo en el que vivimos.

Dicen:

... “soy lesbiana madre, lesbiana política”...

..."Yo soy lesbiana y contrario a la mayoría de las madres heterosexuales, necesito pensar cada día en estrategias para que el patriarcado no lastime a mi familia. Por eso me enuncio, por eso me visiblizo, por eso lucho y por eso existe este blog".

"Soy lesbiana y no puedo separarlo de ser madre porque me atrevo a ejercer una maternidad que a muchas personas les ofende, les cuestiona y les explota sus limitados pensamientos".

Tal como en el imaginario de las izquierdas de los años 70, de los que se trata es de formar "hombres y mujeres nuevos", capaces de revolucionar y sacudir anquilosadas estructuras binarias.

En cuanto a la crianza, señalan que enseñan a sus hijos a poner en duda la heteronormatividad, el falocentrismo y los privilegios masculinos. Dicen:

"Tal vez hasta le sea difícil entender que la vida que le tocó vivir es más fácil porque es hombre. No sabemos cómo ni cuándo se vaya a poner a reflexionar sobre ese privilegio, pero lo que sí podemos asegurar es que puede tener pensamientos feministas".

9. El texto fue leído por primera vez en Nueva York, en la Modem Language Association Convention en 1978, y está dedicado a las lesbianas americanas. A su vez fue publicado por primera vez en Feminist Issues $1, \mathrm{n}^{\circ} 1$, durante el verano 1980 . 
También, esta idea de ver a la crianza como una militancia puede verse en las siguientes entradas:

"En una ocasión, en una marcha, Santiago, de 4 años en aquel entonces, vio a una trans y me preguntó: ¿por qué va así? y le dije, porque así se siente más cómoda para expresar quién es. Santiago a los 4 años lo entendió, se me hace bien complicado que lxs adultxs no lo entiendan. Y que la persona camine desnuda o con lentejuelas por la calle no la hace en nada menos merecedora de respeto".

O también:

"Si la sociedad tiene un problema con la expresión de género de alguna persona, entonces es trabajo de la sociedad el aprender y cambiar. Y yo, la verdad, prefiero llevar a mis hijos a que marchen con las "locas", a llevarlos a una marcha donde sólo aprenderían que lo correcto es "disimular"y vernos "decentes".

Otro:

"Es tan inteligente como cualquier niña. Asegúrate de que lo sepa y se le quede grabado y no ande molestando a las niñas pensando que las puede o sabe más que ellas”.

Por otro lado, el énfasis pedagógico-militante también se lee en los múltiples esfuerzos que relatan en pos de desmontar el pensamiento binario que conduce a que, socialmente, se les cuestione que pueda existir una familia con dos madres; sin figura paterna.

"Me dicen: ¿dos mamás? Si los niños salieron de un solo vientre. La madre verdadera es la biológica, la que se embaraza, la que pare. Esa es la verdadera madre".

Y continúa:

“...en un proyecto de (co)-maternidad esa idea está por completo fuera de la ecuación. La mayoría de ustedes saben que biológica y médicamente yo podría alegar que "soy la verdadera madre"de Santiago, pero me fastidia que ese tenga que ser el argumento para defender el núcleo de convivencia que Ana y yo decidimos formar".

El objetivo político del blog también se ve en el hecho de pensarlo como una herramienta de información y ayuda para madres lesbianas que viven en Méjico. Además de los posteos en los que se narra acerca de la vida cotidiana de esta familia, el blog tiene las siguientes entradas con información:

¿Cómo me puedo casar en el DF?

¿Cómo nacieron Santiago y Diego?

¿Cómo puedo registrar a mi bebé con dos mamás?

¿Dónde y con quién puedo hacer un tratamiento de reproducción asistida en el DF?

¿Qué se necesita para separarse de un pacto civil de solidaridad?

¿Qué se necesita para firmar el pacto civil de solidaridad en Saltillo? 
Un último aspecto que es importante destacar es que los escritos no se limitan a cuestionar los modelos patriarcales de maternidad, familia y heteronormatividad, sino también la monogamia, en tanto que núcleo duro de la familia patriarcal en Occidente.

Así, los posteos registran las experiencias de ambas con múltiples parejas y hacen referencia a que mantienen entre ellas una relación "poliamorosa". Veamos el siguiente posteo:

\section{"COMUNICADO:}

Estimada gente, Criseida Santos Guevara y yo, Ana de Alejandro, queremos hacer de su atento conocimiento lo siguiente: En un momento donde cada reivindicación es política y lo personal es político, hay reivindicaciones MUY personales y por lo tanto MUY políticas que es necesario realizar.

Queremos evitar rumores y especulaciones, acallar voces malvibrosas y prevenir malentendidos, por eso consideramos importante que se haga público que desde 2009 y hasta la fecha mantenemos una relación abierta y poliamorosa.

La decisión de legalizar nuestra unión en el estado civil matrimonial, como es bien sabido, fue principalmente motivada por la necesidad de afianzar y legalizar los lazos de parentesco, social y legalmente entre ambas madres y nuestros hijos, ya que como proyecto de vida común, y prioritario para ambas, lo creímos conveniente para ellos.

Aun así, estados civiles a parte, nos reconocemos la una a la otra como seres libres, independientes, autónomas y capaces de tomar nuestras propias decisiones. (...). Cada una de nuestras cuerpas es única y exclusivamente de cada una de nosotras. Nosotras, cada una, elegimos con quién, cómo, cuándo, dónde y por qué lo compartimos, o no. Reconocemos que nuestro amor y nuestra amistad van más allá de cualquier constructo social monógamoheteronormativo impuesto".

A partir de esa entrada, las autoras se afirman en sus relatos personales para reconocerse como capaces de crear y construir nuevas estructuras, nuevos amores y nuevas formas de encarnar la maternidad.

\section{A MODO DE CIERRE}

Por último, tal como apunta Stone-Mediatore, las "experiencias marginalizadas", es decir, los tipos de experiencias que son sistemáticamente oscurecidas u omitidas en las representaciones dominantes del mundo, están estrechamente relacionadas con posiciones de sujetos económica, política y culturalmente marginalizados/as (Stone-Mediatore, 1999). En esta dirección, podemos decir que las narrativas acerca de las experiencias de maternidad han sido históricamente construidas y gestionadas por varones, arrebatándose a las mujeres y lesbianas la posibilidad de encarnar el lugar de la enunciación acerca de las mismas. Es por eso que, desde los años setenta en adelante, la búsqueda por politizar las experiencias personales de opresión ha guiado las luchas de los feminismos y el movimiento LGBTTTIQ.

En el presente trabajo abordamos lo que nos pareció un fenómeno interesante para estudiar, esto es: la emergencia a partir del uso de plataformas digitales de relatos disidentes en torno a la maternidad, por parte de mujeres y lesbianas, desde un punto de vista feminista. Relatos a partir 
de los cuales hacen oír sus voces rebeldes, escritos que dan cuenta de los modos cómo lo personal deviene político, una vez que se inscribe en el espacio de los discursos públicos.

Paradójicamente, la circulación en los sectores medios de estos relatos disidentes sobre la maternidad, ha sido posible gracias al uso de una tecnológica que se creía exclusivamente al servicio de los intereses patriarcales y coloniales: Internet. Según Zafra (2008) el uso de la web implica una reapropiación del cuarto propio (tradicionalmente identificado como parte de la esfera privada) convirtiéndolo, por tanto, en un cuarto propio conectado a Internet.

Es así como las/es subalternas/es han tomado por asalto las herramientas digitales para ver cómo sus reflexiones se comparten y debaten en las redes. Porque para las mujeres y lesbianas el acto de escribir acerca de sí mismas de manera pública -y no que otros lo hagan por ellas- es un acto de rebeldía ante el silencio impuesto por siglos.

Así, la conquista de un lugar de enunciación que no dependa de la voluntad de las grandes editoriales, ni de la aprobación de ninguna institución ha posibilitado para muchas, no todas decíamos al principio- la posibilidad de difundir públicamente la mirada personal acerca de esta experiencia, y empezar a construir -a contrapelo de los relatos tradicionales- la propia historia. Y eso es, en sí mismo, un acto de transgresión al orden patriarcal. Pues en la producción de estos otros relatos se juega la disputa por el sentido de esas experiencias y la posibilidad de empezar a construir otros mundos posibles.

Y eso implica propiciar la creación y la reproducción de la vida en el terreno de la decisión, la lucha, la sorpresa, la imaginación y la inteligencia consciente (Rich, 1986). Es entender a la maternidad como una tarea libremente elegida (Rich, 1986) y porque no, libremente narrada, a partir de nuestras propias voces.

\section{REFERENCIAS BIBLIOGRÁFICAS}

Andréu Abela, J. (2011). Las técnicas de análisis de contenido: una revisión actualizada. Sevilla: Fundación Centro de Estudios Andaluces. http://public.centrodeestudiosandaluces.es/pdfs/S 200103.pdf

Badinter, E. (1991). ¿Existe el instinto maternal? Historia del amor maternal. Siglos XVII al XX. Barcelona: Paidós.

Boix, M. (2007). Hackeando el patriarcado: La lucha contra la violencia hacia las mujeres como nexo. Filosofía y práctica de Mujeres en Red desde el ciberfeminismo social. Publicado en Mujeres en Red, periódico feminista. Disponible en: www.mujeresenred.net/spip.php?article 880

Carolina Justo en Facebook (2014-2015). www.facebook.com/justovonlurzer

Castaño Collado, C. (2009). La primera y la segunda brecha digital. Revista bibliográfica de geografía y ciencias sociales (Serie documental de Geo Crítica), XIV(825). Universidad de Barcelona.

De Barbieri, T. (1992). Sobre la categoría de género. Una introducción teórica-metodológica. ISIS Internacional, (17). Santiago de Chile. 
Echeverría, G. (2005). Análisis cualitativo por categorías. Apuntes docentes de Metodología de la investigación. Universidad Academia de Humanismo Cristiano. Escuela de Psicología. Disponible en: www.academia.edu/9444115/Apuntes_Docentes_de_METODOLOGIA_DE_IN VESTIGACION_ANALISIS_CUALITATIVO_POR_CATEGORIA

Fernández, A. M. (1999). La mujer de la Ilusión. Pactos y contratos entre Hombres y Mujeres. Buenos Aires: Paidós.

Fernández, A. M. (2013). La diferencia Desquiciada. Géneros y diversidades sexuales. Buenos Aires: Biblos.

García Manso, A. (2007). Cyborgs, mujeres y debates, El Ciberfeminismo como teoría crítica. Revista Castellano-Manchega de Ciencias Sociales. Disponible en: http://dx.doi.org/10.20932/ barataria.v0i8.202

Haraway, D. (1985). Manifiesto Cyborg (trad. M. Talens). Disponible en: https://xenero.webs.uvi go.es/profesorado/beatriz_suarez/ciborg.pdf

Hine, C. (2004). Etnografía virtual. Catalunya, España: Editorial UOC.

Las Dos Mamis, Activismo virtual. (s.d.). Blog. Disponible en: http://dosmamis.blogspot.com.ar/

Luna, R. (2013). Facebook: de la realidad virtual a la virtualidad real. Redes digitales y comunicación: Historia de las plataformas y nuevos vínculos sociales (Sebastián Ramiro Castro Rojas);. - 1a ed. - Rosario: UNR Editora. Disponible en: https://es.calameo.com/books/0026005 $64281 \mathrm{~d} 4965 \mathrm{~b} 512$

Manzanares, G. (2015). Mi protesta está en Internet. Revista Pillku. Recuperado de https://pillku. org/article/mi-protesta-esta-en-internet/

Muñoz Santamaría, L. (2009). Internet, un espacio de empoderamiento para las mujeres. Mujeres en Red. www.mujeresenred.net/spip.php?article1837

Reguillo, R. (2017). \#WomensMarch: redes y calles La búsqueda del futuro. Revista digital Tercera vía. Recuperado de http://terceravia.mx/2017/01/womensmarch-redes-calles-labusqueda-del/

Rich, A. (1986). Nacemos de mujer. La crisis de la maternidad como institución y como experiencia. Barcelona: Noguer.

Scott, J. (1993). El género una categoría útil para el análisis histórico. In C. Cangiano \& L. Dubois, De mujer a género. Buenos Aires: CEAL.

Stone-Mediatore, S. (1999). Chandra Mohanty y la revalorización de la experiencia. Hiparquía, 10(1): 85-107.

Velázquez, L. (2015). Redes Sociales y Activismo Feminista. UNESCO. Gender, Media, ICTs and Journalism - 20 years after the BPfA. www.unesco.org/fileadmin/MULTIMEDIA/HQ/CI/CI/ pdf/panel_1_4_luisa_velazquez_spanish.pdf

Williams, R. (1980). Marxismo y literatura. Barcelona: Península.

Wittig, M. (1973). El cuerpo lesbiano. Valencia: Pre-Textos. 
Relatos disidentes sobre experiencias de maternidad. Una lectura desde el punto de vista feminista...

Wittig, M. (2006). El pensamiento heterosexual y otros ensayos. Barcelona: Editorial EGALES.

Zafra, R. (2008). Un cuarto propio conectado. Feminismo y creación desde la esfera públicoprivada online. Rev. Asparkía, (22), Investigación Feminista. 